\title{
USO DE COLESTEROL NA CRIOPRESERVAÇÃO ESPERMÁTICA E FERTILIDADE: UMA REVISÃO
}

\author{
André Giarola Boscarato \\ Leonardo Franco Martins ${ }^{2}$ \\ Rogério Oliveira Pinho ${ }^{3}$ \\ Gustavo Guerino Macedo ${ }^{4}$ \\ Carla Faria Orlandini ${ }^{1}$
}

BOSCARATO, A. G.; MARTINS, L. F.; PINHO, R. O.; MACEDO, G. G.; ORLANDINI, C. F. Uso de colesterol na criopreservação espermática e fertilidade: uma revisão. Arq. Ciênc. Vet. Zool. UNIPAR, Umuarama, v. 17, n. 2, p. 145-150, abr./ jun. 2014.

RESUMO: A criopreservação é um método amplamente utilizado para o armazenamento de células espermáticas em longo prazo. Essa técnica facilita a difusão e a disponibilidade de material genético a ser utilizado tanto na produção in vitro (PIV) como na inseminação artificial, biotecnologia aplicada na maioria das espécies domésticas. Pesquisas têm desenvolvido estratégias para otimizar a utilização do sêmen por meio da redução das injúrias ocorridas durante o processo de criopreservação, e vem conseguindo melhorar os parâmetros espermáticos pós-descongelação com a adição de ciclodextrina carregada com colesterol (CCC) ao sêmen puro ou previamente diluído das diferentes espécies estudadas. Apesar dos resultados promissores, melhor fertilidade do sêmen tratado com CCC tem sido demonstrada somente in vitro, enquanto que in vivo os resultados ainda não são compensatórios, necessitando maiores investigações para tornar viável a aplicação comercial dessa técnica. O objetivo dessa revisão foi compilar alguns resultados dos principais pesquisadores na área, expondo o estado atual e as perspectivas para melhorias da fertilidade do sêmen tratado com CCC.

PALAVRAS-CHAVE: Ciclodextrina. Inseminação artificial. Membrana plasmática. Semen.

\section{USE OF CHOLESTEROL IN SPERM CRYOPRESERVATION AND FERTILITY: A REVIEW}

\begin{abstract}
Cryopreservation is a method widely used for the long-term storage of sperm cells. This technique facilitates the dissemination and availability of genetic material to be used in both in vitro production (IVP) and in artificial insemination, the biotechnology applied to most domestic species. Research has developed strategies to optimize the use of semen through the reduction of injuries occurred during the cryopreservation process, having succeeded in improving post-thaw sperm parameters with the addition of cholesterol-loaded cyclodextrin (CLC) to pure or previously diluted semen samples from the different species studied. Despite the promising results, better fertility of semen treated with CLC has only been demonstrated in vitro, while in vivo results are not yet feasible, requiring further investigation to result in viable commercial application of such technique. The objective of this review is to compile a few results from the leading researchers in the area, exhibiting the current state and prospects for improving the fertility of semen treated with CLC.
\end{abstract}

KEYWORDS: Cyclodextrin. Artificial insemination. Plasma membrane. Semen.

\section{USO DE COLESTEROL EN LA CRIOPRESERVACIÓN ESPERMÁTICA Y FERTILIDAD: UNA REVISIÓN}

RESUMEN: La criopreservación es un método ampliamente utilizado para el almacenamiento de células espermáticas a largo plazo. Esa técnica facilita la difusión y la disponibilidad de material genético a ser utilizado tanto en la producción in vitro (PIV) como en la inseminación artificial, biotecnología aplicada en la mayoría de las especies domesticas. Investigaciones han desarrollado estrategias para perfeccionar la utilización del semen por medio de la reducción de las injurias ocurridas durante el proceso de criopreservación, y ha conseguido mejorar los parámetros espermáticos pos descongelamiento con la adición de ciclodextrina cargada con colesterol (CCC) al semen puro o previamente diluido. A pesar de los resultados promisores, mejor fertilidad del semen tratado con $\mathrm{CCC}$ ha sido demostrado solo in vitro, mientras que in vivo los resultados todavía no son compensatorios, necesitando de mayores investigaciones para volver viable la aplicación comercial de esa técnica. El objetivo de esa revisión ha sido compilar algunos resultados de los principales investigadores del área, exponiendo el estado actual y las perspectivas para mejorías de la fertilidad del semen tratado con CCC.

PALABRAS CLAVE: Ciclodextrina. Inseminación artificial. Membrana plasmática. Semen.

Introdução

A criopreservação é um método amplamente utili- zado para o armazenamento de células espermáticas a longo prazo. Essa técnica facilita a difusão e a disponibilidade de material genético a ser utilizado tanto na produção in vitro

DOI: https://doi.org/10.25110/arqvet.v17i2.2014.4933

${ }^{1}$ Mestrando do Programa de Mestrado em Ciência Animal - Universidade Paranaense - UNIPAR. andreboscarato@hotmail.com;

${ }^{2}$ Professor Titular do curso de Medicina Veterinária e do Programa de Mestrado em Ciência Animal - Universidade Paranaense - UNIPAR. leonardofmpr@ yahoo.com.br;

${ }^{3}$ Pós doutorando no curso de Zootecnia da Universidade Federal de Viçosa;

${ }^{4}$ Professor Titular do curso de Medicina Veterinária da Universidade Federal de Uberlândia. 
de embriões como na inseminação artificial, biotecnologia aplicada à maioria das espécies domésticas e que vem sendo utilizada há décadas com taxas de concepção comparável à monta natural.

A membrana plasmática é fundamental para a sobrevivência e manutenção da viabilidade da célula espermática no trato reprodutivo feminino, garantindo a homeostase celular e a capacidade fertilizante. Dessa forma, lesões ocorridas nas membranas plasmáticas durante a criopreservação levam a perda de componentes intracelulares, tais como enzimas metabólicas e de ATP (GRAHAM; MOCÉ, 2005) tendo como consequência, prejuízos na motilidade, diminuição da capacidade fertilizante e até a morte celular (CELEGUINI, 2005, GRAHAM; MOCÉ, 2005).

A congelação/descongelação causa na célula choque térmico e osmótico, que induzem a ocorrência de danos parcialmente irreversíveis ao espermatozoide, decorrentes de modificações da membrana plasmática (SOARES; GUERRA, 2009), pela desestabilização da bicamada lipídica que a constitui (WATSON, 2000; MORRIS et al., 2012). Esta desestabilização ocorre à medida que a temperatura é diminuída e a membrana sofre uma transição de fase, a partir da fase fluida para a fase de gel (PURDY; GRAHAM 2004a; GRAHAM; MOCÉ, 2005).

Durante o processo de criopreservação, as membranas espermáticas devem suportar uma variedade de injúrias, o que inclui estresse térmico, mecânico, químico e osmótico (PARKS; GRAHAM, 1992).Assim, a longevidade e a fertilidade do sêmen são reduzidas (MOCÉ; GRAHAM 2006), além da morte de cerca de 10 a $50 \%$ dos espermatozoides, mesmo com protocolos de criopreservação otimizados.Estes fatores são atualmente compensados pela adição de maior número de espermatozoides por inseminação, deposição do sêmen mais próximo ao local da fecundação e próximo ao momento da ovulação (WATSON, 2000). No intuito de melhorar a técnica, muitas estratégias têm sido utilizadas para contornar os pontos críticos dos protocolos de criopreservação, com base no entendimento de quando e onde ocorrem os danos às células espermáticas (MOCÉ et al., 2010).

O objetivo dessa revisão foi compilar alguns resultados dos principais pesquisadores na área, expondo o estado atual e as perspectivas para melhorias da fertilidade do sêmen tratado com ciclodextrina carregada com colesterol.

\section{Desenvolvimento}

\section{Membrana plasmática e colesterol}

Os lipídios que compõem a membrana plasmática dos espermatozoides mamíferos são preferencialmente os insaturados de carga neutra ou positiva, como fosfatidilcolina e esfingomielina na face externa, e os insaturados de carga negativa como fosfatidiletanolamina, fosfatidilserina e fosfatidilinositol, na face interna da bicamada lipídica (LEHNINGER; NELSON; COX, 2005; AMORIM, 2008).O grau de fluidez da membrana depende da temperatura e da relação entre os níveis de ácidos graxos saturados (colesterol) e insaturados. Em temperaturas mais baixas, ocorre pouca movimentação lipídica e a bicamada assume uma forma quase cristalina. Quanto maior a proporção dos ácidos graxos saturados, maior é a temperatura de transição sólido-fluido da membrana (LEHNINGER; NELSON; COX, 2005).

A modificação da composição lipídica das membranas plasmáticas dos espermatozoides afeta a criosobrevivência (AMORIMet al., 2009). Acredita-se que, pelo menos parte das diferenças na sensibilidade dos espermatozoides ao choque térmico esteja relacionada ao seu conteúdo de lipídios. Algumas espécies produzem espermatozoides altamente suscetíveis ao choque térmico (varrão, touro, carneiro e garanhão), outros são menos sensíveis (cão e gato), enquanto outros são altamente resistentes ao choque térmico (coelho, humano e aves) (MOCÉ et al., 2010).Perda de colesterol da membrana plasmática de células criopreservadas tem sido observada em suínos (50\%) e garanhões (28\%) (CEROLINI et al., 2001; MOORE; SQUIRES; GRAHAM, 2005), sendo que esta perda de colesterol pode causar uma capacitação prematura, reduzindo a viabilidade de espermatozoides criopreservados no trato reprodutivo feminino (MOCÉ et al., 2010; BLANCH et al., 2012). O colesterol altera a membrana espermática, protegendo a célula durante o resfriamento. Portanto a adição de colesterol pode ajudar a minimizar ou eliminar a transição de fase durante o processo de resfriamento (GRAHAM; FOOTE; HOUGH, 1987).

O colesterol pode ser facilmente incorporado ou extraído das membranas plasmáticas das células usando ciclodextrinas (MOORE; SQUIRES; GRAHAM, 2005). As ciclodextrinas são oligossacarídeos cíclicos compostos por seis $(\alpha$ - ciclodextrina), sete $(\beta$ - ciclodextrina) ou oito $(\gamma$ - ciclodextrina) unidades de glicopiranose unidas por ligações $\alpha$ $(1,4)$, cujas moléculas se caracterizam por ter uma superfície externa hidrofílica e uma cavidade interna lipofílica (BANCHERO; RONCHETTI; MANNA, 2013).Modificando asciclodextrinas com grupos metil ou hidroxipropilaumenta-sea sua solublidade em água e, consequentemente, a sua capacidade para solubilizar compostos hidrofóbicos (YANCEY et al. , 1996).A metil $\beta$ - ciclodextrina tem sido utilizada por vários pesquisadores, obtendo-se resultados consistentes.

Aumento das taxas de sobrevivência a criopreservação foi encontrado em equinos (COMBES et al. 2000; MOORE; SQUIRES; GRAHAM, 2005;SPIZZIRI et al., 2010), bovinos (PURDY; GRAHAM, 2004b), bisão americano (HUSSAIN; LESSARD; ANZAR, 2013) ovinos (MORRIER et al., 2004, MOCÉ; PURDY; GRAHAM, 2010)caprinos (KONYALI, 2009;AMIDI; FARSHAD; KHOR, 2010;FARSHAD et al. 2011) esuínos (GALANTINO-HOMER et al. 2006, TOMÁS et al. 2013) pela adição de diferentes concentrações de ciclodextrina carregada com colesterol durante o processo de criopreservação, o que permitiu a manutenção de um elevado teor de colesterol na membrana espermática, que por sua vez pode impedir que as células sofram pré-maturação e capacitação precoce, aumentando sua viabilidade.

\section{Preparo da solução de ciclodextrina carregada com co- lesterol (CCC)}

Purdy e Graham (2004a) conduziram um experimento para determinar se o tratamento de sêmen de touros com CCC poderia aumentar a porcentagem de células móveis e viáveis após a congelação, e também para desenvolver um protocolo ótimo de congelação para sêmen bovino tratado com ciclodextrina carregada com colesterol. Para incorporar colesterol à membrana plasmática ao invés de re- 
tirá-lo, o núcleo lipofílico da molécula de ciclodextrina deve estar previamente ocupado pelo composto lipídico em questão, devido ao fato de que as moléculas de colesterol podem difundir-se diretamente a partir da membrana plasmática para o núcleo hidrofóbico da molécula de ciclodextrina, pois essa transferência direta necessita de muito menos energia em comparação ao processo inverso (YANCEY et al., 1996). Sendo assim, Purdy e Graham (2004a) desenvolveram um protocolo para incorporar o colesterol à ciclodextrina que é composto por algumas etapas.

Inicialmente $200 \mathrm{mg}$ de colesterol foram dissolvidos em $1 \mathrm{~mL}$ de clorofórmio.Em um tubo separado, $1 \mathrm{~g}$ de metil$\beta$-ciclodextrina foi dissolvido em $2 \mathrm{~mL}$ de metanol. Uma alíquota de $0,45 \mathrm{~mL}$ da solução de colesterol foi adicionada a solução de ciclodextrina, agitando até a mistura ficar clara, sendo logo em seguida vertida em uma placa de petri de vidro e os solventes evaporados. Os cristais resultantes foram deixados para secagem por mais 24 horas, quando foram retirados da placa e armazenados em um recipiente de vidro a $22^{\circ} \mathrm{C}$.Uma solução de trabalho da ciclodextrina carregada com colesterol foi preparada por adição de $50 \mathrm{mg}$ de CCCa 1 $\mathrm{ml}$ de TALP a $37^{\circ} \mathrm{C}$, misturando a solução brevemente utilizando um misturador tipo vórtex.

A mesma metodologia de preparo da solução de CCC tem sido empregada para adição de colesterol no sêmen equino (MOORE; SQUIRES; GRAHAM, 2005; SPIZZIRI et al., 2010; OLIVEIRA et al., 2010), suíno, (TOMÁS et al, 2011; TOMÁS et al., 2013), ovino (MOCÉ; PURDY; GRAHAM, 2010), em coelhos (SERIN; AKSOY; CEYLAN, 2011) e bovino (MOCÉ; GRAHAM, 2006, AMORIM, 2009; MORAES et al, 2010).

\section{Fertilidade do sêmen tratado com CCC}

O colesterol adicionado é incorporado em todos os compartimentos da membrana plasmática das células espermáticas e faz isso de uma maneira dose dependente aumentando linearmente com a dose de CCC (PURDY; GRAHAM, 2004a). A concentração mais utilizada nos trabalhos revisados fica entre 1 e $2,5 \mathrm{mg}$ para cada $120 \times 10^{6}$ espermatozoides (tabela 1), correlacionando-se negativamente com a fertilidade com concentrações abaixo de $0,5 \mathrm{mg}$ ou acima de 4mg (MOCÉ; PURDY; GRAHAM, 2010).

Tabela 1: Concentração de ciclodextrina carregada com colesterol (CLC) utilizada em diferentes espécies.

\begin{tabular}{lll}
\hline Espécies & Autores & $\begin{array}{l}\text { Concentração/Nú- } \\
\text { mero de esperma- } \\
\text { tozoides }\end{array}$ \\
\hline Bovina & Purdy e Graham (2004a) & $1,5 \mathrm{mg} / 120 \times 10^{6}$ \\
& Purdy et al. (2005) & $1,5 \mathrm{mg} / 120 \times 10^{6}$ \\
& Mocé e Graham (2006) & $2,0 \mathrm{mg} / 120 \times 10^{6}$ \\
& Amorim et al. (2009) & $1,5 \mathrm{mg} / 120 \times 10^{6}$ \\
& Moraes et al. (2010 & $1,5 \mathrm{mg} / 120 \times 10^{6}$ \\
& Zahnet al. (2002) & $0,125 \mathrm{mM}$ \\
& Moore et al. (2005) & $1,5 \mathrm{mg} / 120 \times 10^{6}$ \\
& Spizziriet al. (2010) & $1,5 \mathrm{mg} / 120 \times 10^{6}$
\end{tabular}

\begin{tabular}{lll} 
& Oliveira et al. (2010) & $1,5 \mathrm{mg} / 120 \times 10^{6}$ \\
\multirow{4}{*}{ Ovino } & Combeset al. (2010) & $0,125 \mathrm{mmolchol} / \mathrm{L}$ \\
& Mocéet al. (2010) & $2 \mathrm{mg} / 120 \times 10^{6}$ \\
& Purdyet al. (2010) & $2 \mathrm{mg} / 120 \times 10^{6}$ \\
& deGraaf et al. (2007) & $1,8 \mathrm{mg} / 120 \times 10^{6}$ \\
Caprino & Amidi; Farshad; Khor & $1,5 \mathrm{mg} / 240 \times 10^{6}+$ \\
& $(2010)$ & $\mathrm{BSA}^{* *} 2,5 \%$ \\
& Farshadetal.,(2011) & $2,25 \mathrm{mg} / 240 \times 106$ \\
& Koniyali (2009) & $1 \mathrm{mg} / 120 \times 106$ \\
Suíno & Zeng e Terada (2001) & $20 \mathrm{mM} \mathrm{MBC} *$ \\
& & $+10 \mathrm{mM}$ \\
& Tomás et al. (2009) & $1,0 \mathrm{mg} / 120 \times 10^{6}$ \\
& Tomás et al. (2013) & $1,0 \mathrm{mg} / 120 \times 10^{6}$ \\
\hline
\end{tabular}

*MBC $=$ metil- $\beta$-ciclodextrina.

Todas as espécies avaliadas quanto à adição de CCC à membrana espermática responderam positivamente com aumento da viabilidade pós-descongelação, sendo os parâmetros mais favorecidos a motilidade e integridade de membrana (COMBESet al., 2000; PURDY; GRAHAM, 2004a; MOCÉ; PURDY; GRAHAM, 2010; HUSSAIN; LESSARD; ANZAR, 2013), resistência ao choque osmótico (MOCÉ; PURDY; GRAHAM, 2010; TOMÁS et al., 2011), correlação positiva com a fertilidade in vitro (MOORE; SQUIRES; GRAHAM, 2005; SPIZZIRI et al., 2010; TOMAS et al 2011; MOCÉ; PURDY; GRAHAM, 2010).

Resultados positivos foram encontrados nos parâmetros espermáticos pós-descongelação em várias espécies, porém estes não se refletem positivamente na fertilidade, que apresenta resultados ainda inconsistentes (tabela 2).

Tabela 2: Efeito do tratamento do sêmen com ciclodextrina carregada com colesterol (CCC) sobre a capacidade fertilizante do sêmen in vivo.

\begin{tabular}{lccc}
\hline Espécies & $\begin{array}{c}\text { Dose/Inseminação } \\
\text { artificial }\end{array}$ & Controle & $\begin{array}{c}\text { Tratado } \\
\text { com CCC }\end{array}$ \\
\hline${ }^{\text {a} B o v i n a ~}$ & 750000 & $50 \%$ & $59 \%$ \\
${ }^{\mathrm{b}}$ Equina & $800 \times 10^{6}$ & $75 \%$ & $25 \%$ \\
${ }^{\mathrm{c} E q u i n a}$ & $400 \times 10^{6}$ & $28,6 \%$ & $15 \%$ \\
${ }^{\mathrm{d}}$ Ovina & $100 \times 10^{6}$ & $45,3 \%$ & $41 \%$ \\
${ }^{\mathrm{e} S u i ́ n a *}$ & $600 \times 10^{6}$ & $67,7 \%$ & $55,9 \%$ \\
${ }^{\mathrm{e}}$ Suína** & $600 \times 10^{6}$ & $65,2 \%$ & $66,7 \%$ \\
\hline
\end{tabular}

${ }^{\mathrm{a} P u r d y}$ and Graham (2004b); ${ }^{\mathrm{b}} \mathrm{Zahn}$ et al. (2002); 'Spizziri et al. (2010); 'Purdy et al. (2010); ${ }^{\text {CT}}$ Tomás et al. (2013).* IA 37 horas após indução da ovulação com hCG; **IA 30 horas após indução da ovulação com hCG.

Adaptado de Mocé et al. (2010).

Em equinos, Oliveira et al. (2010) sugeriram que a incorporação de colesterol à membrana plasmática atrasa os processos de capacitação espermática e reduz a reação acrossômica subsequente, o que aumenta o tempo necessário para a capacitação in vivo (SPIZZIRI et al., 2010), fato que prejudicaria a fertilidade, e fundamenta as baixas taxas de gestação (25\%/75\% e $28,6 / 15 \%$ )em éguas inseminadas com e sem a adição de colesterol ao sêmen, respectivamente (ZANH et 
al., 2002; SPIZZIRI et al., 2010). Portanto os espermatozoides tratados com CCC podem precisar ser inseminados em um ponto de tempo diferente em relação à ovulação, permitindo uma capacitação mais longa e reação acrossômicaadequada (SPIZZIRIet al., 2010). Porém foi demonstradoin vitro o aumento (MOORE; SQUIRES; GRAHAM, 2005) ou manutenção (SPIZZIRIet al., 2010) da capacidade de espermatozoides equinos tratados com CCC em se ligar à zona pelúcida de oócitos, fato que é correlacionado positivamente com a fertilidade (MEYERS et al., 1996), e é explicado pela suposição de que os espermatozoides que tenham sido tratados com CCC são protegidos contra os danos agudos da membrana e/ou perda de receptores de espermatozoides/ovócito que ocorre durante a criopreservação (MOORE; SQUIRES; GRAHAM, 2005).

No entanto, nenhum ensaio in vitro calcula com precisão o potencial in vivo de fertilização dos espermatozoides (GRAHAM; MOCÉ, 2005).

Em contraste, no sêmen bovino os espermatozoides tratados ou não com CCC são equivalentes, tanto na sua capacidade de fertilização como no tempo necessário para capacitação (PURDY; GRAHAM, 2004a).Purdy e Graham (2004a) não verificaram diferenças in vitro nas taxas de clivagem e blastocisto, e também in vivo, com taxas de gestação de 59 e $50 \%$, com e sem tratamento com CCC respectivamente.Isto sugere que o aumento do número de espermatozoides que sobrevivem após o congelamento (PURDY; GRAHAM, 2004b; MOCÉ; GRAHAM, 2006; AMORIM et al.,2009) pode melhorar as taxas de fertilidade ou permitir um número menor de espermatozoides utilizados por dose inseminante, sem reduzir a fertilidade, sendo necessário maiores ensaios para confirmação desta hipótese (PURDY; GRAHAM, 2004a).

A adição de CCC ao sêmen suíno criopreservado tem sido altamente benéfica em termos de integridade de acrossoma e parâmetros de motilidade (ZENG; TERADA, 2001). Tomás et al. (2011) não mostraram melhora na qualidade espermática após a criopreservação, mas determinaram que o tratamento do sêmen suíno com CCC aumenta os limites de tolerância osmótica e não modifica a resposta da célula espermática às condições capacitantes, além de uma capacidade superior de penetração no oócito in vitro. Quando inseminados no intervalo de 30 horas após indução da ovulação, as taxas de concepção e parto foram melhores em relação ao grupo controle, podendo-se obter resultados aceitáveis com uma única inseminação realizada neste intervalo, que é o mesmo recomendado quando se utiliza sêmen fresco (TOMÁS et al., 2013). Isto se deve, provavelmente, ao fato de que o aumento dos níveis de colesterol na membrana espermática diminui os efeitos da criopreservação que induzem a capacitação prematura e reduzem a viabilidade. Assim, a inseminação com o intervalo convencionalmente utilizado em suínos (30 horas) permite a capacitação e reação acrossômicaadequadas, resultando em melhores taxas de fecundação, sem alterar a rotina de inseminação, o que torna o sêmen criopreservadocada vez mais atrativo para a indústria de suínos (TOMÁS et al.,2013).

Atualmente, em ovinos e caprinos, fertilidade aceitável usando espermatozoides criopreservados só é conseguida com utilização de inseminação intrauterina por laparoscopia (MOCÉ; PURDY; GRAHAM, 2010), uma técnica que tem limitações devido ao tempo e experiência técnica exigidos além de custos associados (PURDY et al., 2010).A obtenção de melhores parâmetros espermáticos pós descongelação poderiam permitir maiores taxas de fertilidade, possibilitando o uso de técnicas mais práticas. Nestes animais, a adição de CCC tem obtido resultados similares as outras espécies, tanto com adição ao sêmen já diluído (PURDY et al., 2010; MOCÉ; PURDY; GRAHAM, 2010, AMIDI; FARSHAD; KHOR, 2010; FARSHAD et al., 2011) como com adição ao semen puro e posterior diluição (KONYALI, 2009; MOCÉ; PURDY; GRAHAM, 2010).

Em caprinos, adição de CCC ao sêmen antes da congelação melhorou as taxas de motilidade, viabilidade, integridade acrossômica e longevidade dos espermatozoides após o congelamento e descongelamento (KONYALI, 2009; AMIDI; FARSHAD; KHOR, 2010; FARSHAD et al., 2011), porém não há relatos sobre a fertilidade in vitro ou in vivo.

O tratamento com 2-hidroxipropil- $\beta$-ciclodextrina carregada com colesterol manteve maiores níveis de colesterol na membrana plasmática de espermatozoides ovinos após a criopreservação, alcançando melhora na motilidade, integridade de membrana (MORRIER 2004; MOCÉ; PURDY; GRAHAM, 2010; PURDY etal., 2010) e tolerância ao choque osmótico, além de superior longevidade e capacidade in vitro de ligação a zona pelúcida de oócitos(MOCÉ; PURDY; GRAHAM, 2010), fato que, como já mencionado, não possui precisão para determinar o potencial de fertilização in vivo(GRAHAM; MOCÉ, 2005), estando de acordo com resultados encontrados por Purdy et al. (2010), onde o tratamento com CCC não afetou a fertilidade in vivo em ovelhas inseminadas.

Melhores parâmetros espermáticos com a utilização deciclodextrina carregada com colesterol são decorrentes da maior estabilidade de membrana, inibindo assim os efeitos deletérios da criopreservação, sendo um deles a capacitação espermática precoce. No entanto, estudos adequados para determinar se o tratamento com CCC influencia no tempo de capacitação in vivo não foram realizados (MOCEet al.,2010).

\section{Considerações finais}

A incorporação de colesterol à membrana espermática é uma forma eficiente de aumentar a viabilidade, as taxas de sobrevivência, a longevidade e a qualidade dos parâmetros espermáticos do sêmen após o descongelamento. A possibilidade da adição de CCC ao sêmen sem a necessidade de modificação de protocolos convencionais de criopreservação é um atrativo a mais para a difusão desta tecnologia. Porém, para esse método tornar-se uma realidade na prática reprodutiva, melhores taxas de fertilidade necessitam ser alcançadas. A razão pela qual o aumento da qualidade do sêmen não se traduz em uma maior capacidade de fertilização ainda não é conhecida e são necessários mais estudos para sua determinação. Devido a influência do colesterol sobre a capacitação espermática e reação acrossômica, devem ser desenvolvidos protocolos de inseminação específicos para o sêmen de diferentes espécies submetidos a este tratamento, uma vez que exigem maior tempo para capacitação no aparelho reprodutor feminino e, portanto, os baixos índices de fertilidade podem ser resultado de uma inadequada sincronia entre a inseminação dos espermatozoides e a ovulação. 


\section{Referências}

AMIDI, F.; FARSHAD, A.; KHOR, A. K. Effects of cholesterol-loaded cyclodextrin during freezing step of cryopreservation with TCGY extender containing bovine serum albumin on quality of goat spermatozoa. Cryobiology, v. 61, p. 94-99, 2010.

AMORIM, E. A. M. Alteração da membrana espermática de suínos, bovinos e equinos na qualidade do sêmen. 2008. Tese (Doutorado - Programa de Pós-Graduação em Zootecnia) - Universidade Federal de Viçosa.

AMORIM, E. A. M. et al. Effect of cholesterol or cholesterylconjugateson the cryosurvival of bull sperm. Cryobiology, v. 58, p. 210-214, 2009.

BANCHERO, M.; RONCHETTI, S.; MANNA, L. Characterization of Ketoprofen/Methyl- $\beta$ Cyclodextrincomplexes prepared using supercritical carbon dioxide. Journal of Chemistry, v. 2013, p. 1-8, 2013.

BLANCH, E. et al. Response of boar spermto the treatment with cholesterol-loaded cyclodextrins added prior tocryopreservation. Reproduction in Domestic Animals, v. 47, p. 959-964, 2012.

\section{CELEGHINI, E.C.C. Efeitos da criopreservação} do sêmen bovino sobre as membranas plasmática, acrossomal e mitocondrial e estrutura da cromatina dos espermatozoides utilizando sondas fluorescentes. 2005. Tese (Doutorado) - Faculdade de Medicina Veterinaria e Zootecnia, Departamento de Reprodução Animal, Universidade de São Paulo, São Paulo, 2005.

CEROLINI, S. et al. Changes in sperm quality and lipid composition during cryopreservationof boar semen. Reproduction, v. 121, p. 395-401, 2001.

COMBES, G. B. et al. Effect of cholesterol on the motility and plasma membrane integrity of frozen equine spermatozoa after thawing. Journal of Reproduction and Fertility, v. 56, p. 127-132, 2000.

DE GRAAF, S. P. et al. The influence of antioxidant, cholesteroland seminal plasma on the in vitro quality of sorted and non-sortedram spermatozoa. Theriogenology, v. 67, p. 217-227, 2007.

FARSHAD, A. et al. Effect of cholesterol-loadedcyclodextrin in presence and absence of egg yolk during freezing step on quality of Markhoz Buck's spermatozoa. Asian-Australasian Journal of Animal Sciences, v. 24, p. 181-189, 2011

GALANTINO-HOMER, H. L. et al. Effects of 2-hydroxypropylbeta-cyclodextrin and cholesterol on porcine sperm viabilityand capacitation status following cold shock or incubation. Molecular Reproduction and Development, v. 73, p. 638-650, 2006.
GRAHAM, J. K.; FOOTE, R. H.; HOUGH, S. R.

Penetration of zona-free hamster eggs by liposome- treated sperm from the bull, ram, stallion, and boar. Biology of Reproduction, v.37, p.181-188, 1987.

GRAHAM, J. K.; MOCÉ, E. Fertilityevaluation of frozen/ thawed semen.Theriogenology, v. 64, p. 492-504, 2005.

HUSSAIN, S. A.; LESSARD, C.; ANZAR, M. A strategy for improvement of postthaw quality of bison sperm.

Theriogenology, v. 79, p. 108-115, 2013.

KONYALI, C. Effect of cholesterol-loaded cyclodextrinson buck sperm quality after cryopreservation with different extenders. 2009. Tese (Mestrado) - Universidad Polictecnica de Valencia, Valencia, 2009.

LEHNINGER, A. L.; NELSON, D. L.; COX, M. M. Lehninger: Princípios de Bioquímica. 4. ed. São Paulo: Sarvier. 2005. 1120 p.

MEYERS, S. A. et al. Zona pellucida binding and zonainduced acrosome reactions in horse spermatozoa: comparisons between fertile and subfertile stallions. Theriogenology, v. 46, p. 1268-1277, 1996.

MOCÉ, E.; GRAHAM, J. K. Cholesterol-loaded cyclodextrins added to fresh bull ejaculates improve sperm cryosurvival. Journal of Animal Science, v.84, p. 826-833, 2006.

MOCÉ, E. et al. Use of cholesterol in sperm cryopreservation: present moment and perspectives to future. Reproduction in Domestic Animals, v. 45, p. 57 66,2010

MOCÉ, E.; PURDY, P. H.; GRAHAM, J. K.Treating ram sperm with cholesterol-loaded cyclodextrins improves cryosurvival. Animal Reproduciton Science, v. 118, p. 236-247, 2010.

MOORE, A. I.; SQUIRES, E. L.; GRAHAM, J. K. Adding cholesterol to the stallion sperm plasma membrane improves cryosurvival. Cryobiology, v. 51, p. 241-249, 2005.

MORAES, E. A. et al. Delivering cholesterol or cholestanol to bull sperm membranes improves cryosurvival. Animal Reproduction, v. 118, p. 148-154, 2010.

MORRIER, A. et al. Effect of cholesterol loaded methylb-cyclodextrin on ram sperm during cryopreservation, cold-shock and artificial insemination. In: Proceedings of the Society for the Study of Reproduction Meeting. Vancouver, Canada, Abstract 636, p. 239, 2004.

MORRIS, G. J. et al. Freezing injury: The special case of the sperm cell. Cryobiology, v. 64, p. 71-78, 2012.

OLIVEIRA, C. H. et al. Cholesterol addition protects 
membrane intactness during cryopreservation of stallion sperm. Animal Reproduciton Science, v. 118, p. 194-200, 2010 .

PARKS, J. E.; GRAHAM, J. K. Effects of cryopreservation procedures on sperm membranes. Theriogenology, v. 38, p. 209-222, 1992.

PURDY, P. H.; GRAHAM, J. K. Effect of cholesterolloaded cyclodextrin on the cryosurvival of bull sperm. Cryobiology, v.48, p. 36-45, 2004a.

PURDY, P. H.; GRAHAM, J. K. Effect of adding cholesterol to bull sperm membranes on sperm capacitation, the acrosome reaction, and fertility. Biology of Reproduction, v. 71, p. 522-527, 2004b.

PURDY, P. H.; FOX, M. H.; GRAHAM, J. K. The fluidity of Chinese hamster ovary cell and bull sperm membranes after cholesterol addition. Cryobiology, v. 51, p. 102-112, 2005.

PURDY, P. H. et al. The fertility of ram sperm held for $24 \mathrm{~h}$ at 5 degrees $\mathrm{C}$ prior to cryopreservation. Animal

Reproduction Science, v. 118, p. 231-235, 2010.

SERIN, I.; AKSOY, M.; CEYLAN, A. Cholesterol-loaded cyclodextrin inhibits premature acrosomal reactions in liquid-stored rabbit spermatozoa. Animal Reproduction Science, v. 123, p. 106-111, 2011.

SOARES, A. T.; GUERRA, M. M. P. Efeitos da criopreservação sobre a viabilidade espermática. Tecnologia e Ciências Agropecuárias, v. 3, p. 53-63, 2009.

SPIZZIRI, B. E. et al. Cholesterol-loaded-cyclodextrins and fertility potential of stallions spermatozoa. Animal Reproduction Science, v. 118, p. 255-264, 2010.

TOMÁS, C. et al. Cholesterol quantification during cryopreservation in boar sperm samples enriched in or deprived of cholesterol. In: KUBISCH, H. M.; TIAN, X. C. (Eds.). ANNUAL CONFERENCE OF THE INTERNATIONAL EMBRYO TRANSFER SOCIETY (IETS), REPRODUCTION, FERTILITY AND DEVELOPEMENT, 35., Proceeding... San Diego, CA (USA), v. 21, p. 140, 2009.

TOMÁS, C. et al. Treating boar sperm with cholesterol loaded cyclodextrins widens the sperm osmotic tolerance limits and enhances the in vitro sperm fertilizing ability. Animal Reproduction Science, v. 129, p. 209-220, 2011.

TOMÁS, C. et al. In vivo fertilising ability of frozen-thawed boar sperm treated with cholesterol-loaded cyclodextrins prior to cryopreservation. Animal Reproduction Science, v. 140, p. $77-82,2013$.

WATSON, P. F. The causes of reduced fertility with cryopreserved semen. Animal Reproduction Science, v. 60-61, p. 481-492, 2000.
YANCEY, P. G. et al. Cellular cholesterol efflux mediated by cyclodextrins: Demonstration of kinetic pools and mechanism of efflux. Journal of Biological Chemistry, v. 271, p. 16026-16034, 1996.

ZAHN, F. S.; PAPA, F. O.; DELL'AQUA, J. A. Cholesterol incorporation on equine sperm membrane: effects on post thaw sperm parameters and fertility. Theriogenology, v. 58, n. 2, p. 237-240, 2002.

ZENG, W. X.; TERADA, T. Effects of methyl-betacyclodextrin on cryosurvival of boar spermatozoa. Journal of Andrology, v. 22, p. 111-118, 2001.

Recebido em: 30/01/2014 Aceito em: 27/08/2014 